\title{
Search for heavy neutrinos with the ATLAS detector
}

\section{Federico Scutti*}

On behalf of the ATLAS collaboration

The University of Melbourne, Australia

E-mail: federico.scuttidunimelb.edu.au

Multiple theories beyond the Standard Model predict the existence of heavy neutrinos, such as the Type I or Type III See-Saw mechanisms which can explain the light neutrino masses, or LeftRight Symmetric Models which restore parity symmetry in weak interactions at higher energy scale and predict right-handed counterparts to the weak gauge bosons. Searches for such heavy Majorana or Dirac neutrinos with the ATLAS detector, which can also lead to boosted or also displaced signatures, are illustrated using proton-proton data from the LHC at a center-of-mass energy of $13 \mathrm{TeV}$.

The 21st international workshop on neutrinos from accelerators (NuFact2019) August 26 - August 31, 2019

Daegu, Korea

\footnotetext{
*Speaker.
} 
The observation of neutrino oscillations [1] implies that neutrinos are massive, requiring the addition to the Standard Model of particle physics (SM) of a mass-generation mechanism for these light particles. A widely adopted approach to explain small neutrino masses is the so-called SeeSaw mechanism [2], where the light neutrinos acquire their Majorana masses from dimension-5 operators through electroweak symmetry breaking. See-Saw mechanisms can be categorised into a few different types, denominated Type-I [3], Type-II [4] and Type-III [5] scenarios. Models based on Type-I and Type-II scenarios can further be embedded into so-called Left-Right Symmetric Models (LRSM) [6] introduced to explain the broken parity symmetry of the SM. This contains heavy neutrinos $N_{\mathrm{R}}$ which are parity gauge partners of the corresponding left-handed neutrino fields in the SM, and right-handed gauge bosons $W_{R}$. In the LRSM left-handed neutrinos and right-handed neutrinos are considered to be Majorana particles allowing lepton-number violating processes to occur. In LRSM variants, including the inverse See-Saw mechanism [7], $N_{\mathrm{R}}$ neutrinos are pseudo-Dirac particles. For simple versions of the LRSM containing the inverse See-Saw mechanism, lepton-number violating processes are not expected to occur. While Type-I models introduce the $N_{\mathrm{R}}$ singlet, Type-III models introduce two extra triplets of heavy fermionic fields which couple to SM gauge bosons and generate neutrino masses through Yukawa couplings to the Higgs bosons and neutrinos.

In these proceedings, results from recent searches for the heavy right-handed neutrinos $N_{\mathrm{R}}$ are illustrated together with searches for the heavy leptons predicted by Type-III See-Saw models $N^{0}, L^{ \pm}$. These are performed using data collected by the ATLAS [8] experiment based on highenergy proton-proton collisions delivered by the Large Hadron Collider (LHC) [9] at a centreof-mass energy of $\sqrt{s}=13 \mathrm{TeV}$. Different searches are based on different integrated luminosities collected during the Run-II phase of LHC operation and range between $\approx 33 \mathrm{fb}^{-1}$ and $\approx 80 \mathrm{fb}^{-1}$.

A search for right-handed Majorana neutrinos has been performed [10] by looking for a production mechanism where they mix with $v_{\mu}$ or $v_{e}$ which are coupled to $W$ bosons, in a final state with leptonic $W$ decays.

The free parameters of the minimal Type-I model considered are the mass of the heavy neutrino, $m_{\mathrm{N}}$, and the coupling strength $|U|^{2}$, where $U$ is the mixing matrix between $N_{\mathrm{R}}$ and the different light neutrino flavours. This scenario allows for lepton-number conservation (LNC) or violation (LNV). The $N_{\mathrm{R}}$ lifetime has a strong dependence on the coupling strength and the mass due to phase-space effects. Hence, the search is conducted using prompt and displaced leptonic decay signatures. The mass range $5 \mathrm{GeV}<m_{\mathrm{N}}<50 \mathrm{GeV}$ is probed using the prompt signature and assuming LNV. The range $4.5 \mathrm{GeV}<m_{\mathrm{N}}<10 \mathrm{GeV}$, corresponding to decay lengths of the order of mm-cm, is probed down to lower $|U|^{2}$ values using the displaced signature with $N_{\mathrm{R}}$ mixing to $v_{\mu}$ without any assumption regarding LNV or LNC.

The prompt signature requires three leptons produced at the interaction point, either $\mu^{ \pm} \mu^{ \pm} e^{\mp}$ or $e^{ \pm} e^{ \pm} \mu^{\mp}$, with a veto on topologies containing same-flavour and opposite-charge pairs. A requirement is imposed on the three-lepton invariant mass to be within $40 \mathrm{GeV}$ and $90 \mathrm{GeV}$. This search is performed using $36.1 \mathrm{fb}^{-1}$ of data collected by ATLAS.

The displaced signature comprises a prompt muon from the $W$ boson decay and the requirement of a di-lepton vertex, either $\mu \mu$ or $e \mu$ displaced in the transverse plane by $4-300 \mathrm{~mm}$ from the interaction point. A requirement is applied on the invariant mass associated with the two tracks stemming from the displaced vertex to be above $4 \mathrm{GeV}$. The search is conducted with $32.9 \mathrm{fb}^{-1}$ of 
ATLAS data. The so-called large-radius tracking algorithms [11] are used which are computationally expensive and motivate the reduced dataset.

Exclusion limit are shown in Figure 1. The displaced search shows a weaker exclusion limit under the LNV interpretation. This is due to a reduction in the lifetime by a factor of two when LNV decays are allowed.
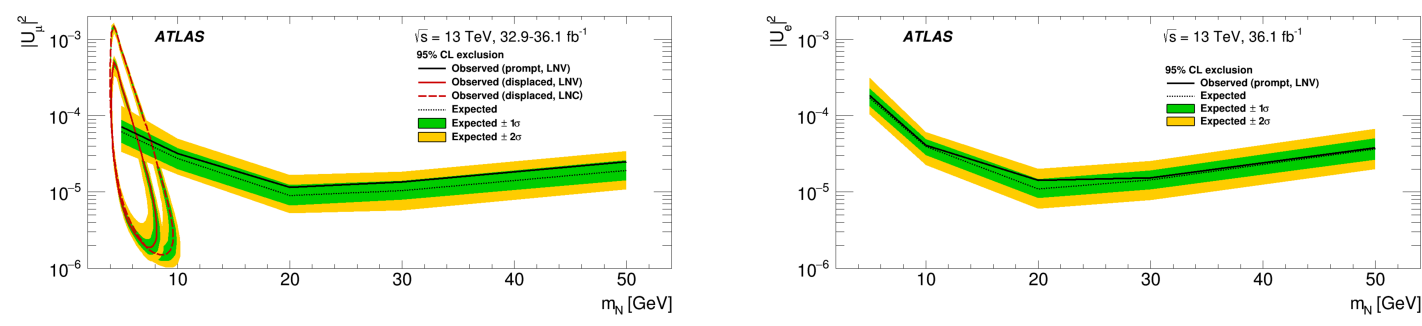

Figure 1: Exclusion limits at 95\% CL in the parameter space of the coupling strength versus the heavy neutrino mass for the search using purely leptonic $W$ boson decays. The left plot illustrates the coupling exclusion in the muon sector while the right plot in the electron one. The region above the black line is excluded for the prompt signature search, while the area enclosed within the red line is excluded by the displaced signature. The solid red lines show limits assuming that $50 \%$ of the decays are LNV and the long-dashed red line the case where they are LNC. Plots are taken from Ref. [10].

A search for pair production of heavy neutral leptons as predicted by Type-III See-Saw models is performed with $79.8 \mathrm{fb}^{-1}$ of ATLAS data. The analysis [12] focuses on $N^{0}$ and $L^{ \pm}$decays with intermediate $W$ bosons and yielding two final state light-leptons which are electrons or muons of different flavour and charge combinations with at least two jets and large missing transverse momentum in the final state. Neutral and charged heavy lepton states are considered mass-degenerate and their mass $m\left(N^{0}, L^{ \pm}\right)$is the only free parameter considered for this model. The unknown mixing angles between the SM leptons and the new heavy lepton states enter only in the expression of the $N^{0}$ and $L^{ \pm}$widths. The production cross-section does not depend on the mixing angles as heavy leptons are produced through couplings to electroweak bosons. Only the branching fractions of heavy leptons decays to a certain lepton flavour depends on the mixing angles. In the analysis these branching ratios are considered to be equal for all three leptonic flavours and fixed to a value of $1 / 3$.

The search is optimised in six channels distinguished by flavour combination and charge product of the final state lepton pair, where same-charge or opposite-charge pairs are considered. A large missing transverse energy significance is required of $\operatorname{Sig}\left(E_{\mathrm{T}}^{\mathrm{miss}}\right)>10$ for opposite-charge final states and $\operatorname{Sig}\left(E_{\mathrm{T}}^{\mathrm{mis}}\right)>7.5$ for same-charge ones. This variable is calculated using a maximum likelihood ratio considering the direction of $E_{\mathrm{T}}^{\text {miss }}$ and the calibrated objects as well as their respective resolutions. The invariant mass of the two leading jets in the event is required to be between $60 \mathrm{GeV}$ and $100 \mathrm{GeV}$. A boosted decay topology is exploited for decay products stemming from heavy leptons, requiring the combined transverse momentum for di-jet and lepton pairs to be above $100 \mathrm{GeV}$. The final exclusion limit is shown in Figure 2.

Heavy right-handed Majorana or Dirac neutrinos $N_{\mathrm{R}}$ are searched for together with the heavy right-handed gauge bosons predicted in the Left-Right Symmetric Model. The search [13] is performed using a pair of two energetic electrons or muons in the final state which have either the 


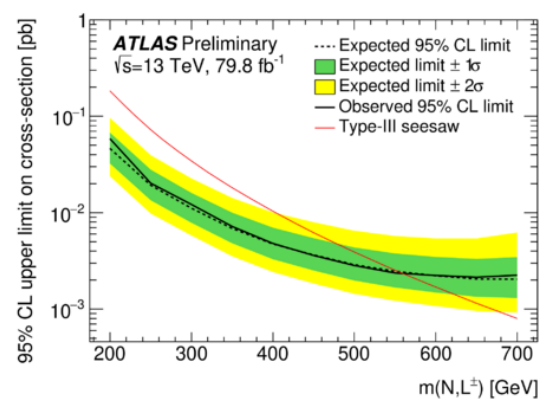

Figure 2: Exclusion limits at 95\% CL for the Type-III See-Saw heavy lepton search. The model crosssection is shown in red. The plot is taken from Ref. [12].

same electric charge or opposite. Different charge combinations are used in independent selections which are also optimised separately for electrons and muons. An integrated luminosity of $36.1 \mathrm{fb}^{-1}$ is used. The masses of the heavy neutrino and of $W_{\mathrm{R}}$ are the free parameters considered. No flavour mixing is considered. In addition to this left-handed and right-handed weak gauge couplings are defined to be equivalent.

The final state contains two charged leptons and two jets. The production mechanism is the so-called Keung-Senjanović (KS) process [14]. When the $W_{\mathrm{R}}$ mass is heavier than the $N_{\mathrm{R}}$ mass the on-shell $W_{\mathrm{R}}$ mass is reconstructed from the invariant mass of the di-lepton and di-jet system, while when the neutrino is heavier only the di-jet mass is considered. The scalar sum between the transverse momenta of the selected leptons and the jets has to be larger than $400 \mathrm{GeV}$.

The Majorana or Dirac nature of $N_{\mathrm{R}}$ is established by comparing the charges of the two leptons in the final state. If $N_{\mathrm{R}}$ is a pseudo-Dirac particle the leptons will always have opposite charge. However, if they are Majorana particles, the final state will contain opposite-charge and same-charge pairs with equal probability. Final exclusion plots are shown in Figure 3 under the assumption of Majorana $N_{\mathrm{R}}$. Corresponding plots for the pseudo-Dirac hypothesis yield similar results due to the similar sensitivities achieved by the opposite-charge and same-charge selection optimisations.
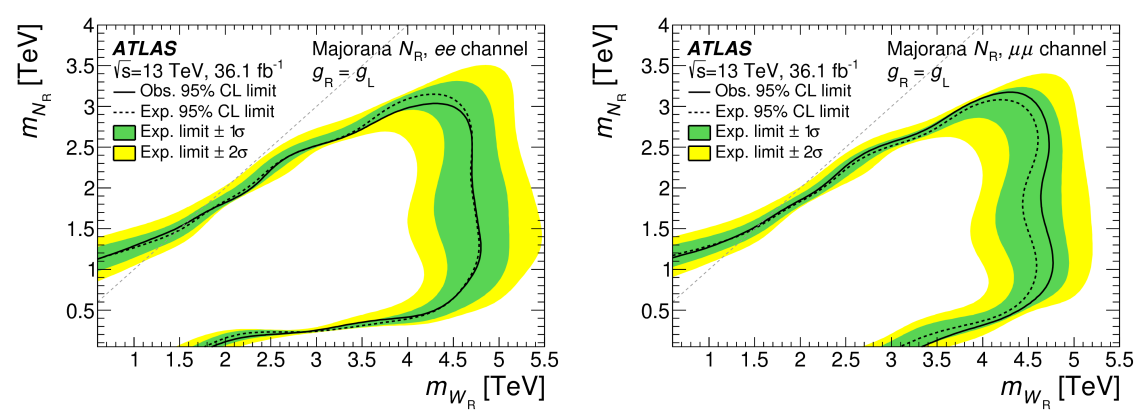

Figure 3: Exclusion limits from the search for heavy Majorana or Dirac neutrinos in the Left-Right Symmetric Model. The area within the curves is excluded at 95\% CL. These limits refer to the hypothesis where the heavy neutrino is a Majorana particle. The dashed gray line indicates the region of the plane where $N_{\mathrm{R}}=W_{\mathrm{R}}$. The plots are taken from Ref. [13].

A different phase space is probed by the search for $W_{\mathrm{R}}$ with boosted $N_{\mathrm{R}}$ decays [15]. Elec- 
tron or muon pairs are considered but no charge-product requirement is applied to the them. An integrated luminosity of $80 \mathrm{fb}^{-1}$ is used. Jets are reconstructed using a large radius algorithm, with radius parameter $R=1.0$, where the two jets in the final state are merged into one which is required to overlap with one light lepton, have a transverse momentum higher than $200 \mathrm{GeV}$, and be backto-back in the transverse plane with respect to one recoiling lepton. Exclusion limits are shown in Figure 4.
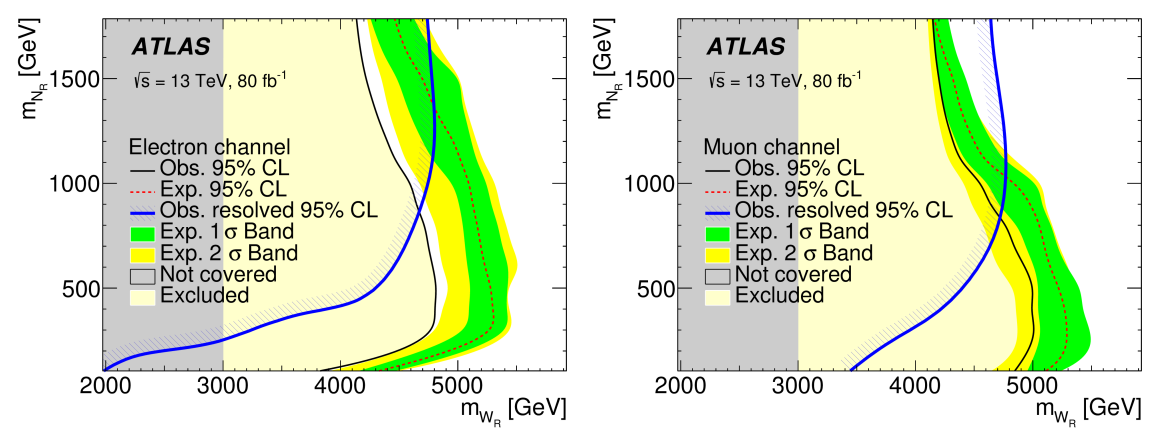

Figure 4: Exclusion limits at $95 \% \mathrm{CR}$ in the boosted decay topology search for $N_{\mathrm{R}}$ and $W_{\mathrm{R}}$ in the LRSM. The blue line illustrates the comparison with the resolved topology search where two jets are required in the final state. The plots are taken from Ref. [15].

No evidence for new physics is reported from these searches performed with the ATLAS detector at the LHC, while they do improve upon previous results.

\section{References}

[1] C. Patrignani et al., Chin. Phys. C40 (2016) 100001.

[2] R. N. Mohapatra and G. Senjanovic, Phys. Rev. Lett. 44 (1980) 912.

[3] T. Yanagida, Conf. Proc. C7902131 (1979) 95.

[4] M. Magg and C.Wetterich, Phys. Lett. B 94 (1980) 61.

[5] R. Foot, H. Lew, X. G. He, and G. C. Joshi, Z. Phys. C44 (1989) 441.

[6] G. Senjanović and R. N. Mohapatra, Phys. Rev. D 12 (1975) 1502.

[7] A. G. Dias, C. A. de S. Pires, P. S. Rodrigues da Silva and A. Sampieri, Phys. Rev. D 86 (2012) 035007.

[8] ATLAS Collaboration, JINST 3 (2008) S08003.

[9] L. Evans and P. Bryant, JINST 3 (2008) S08001.

[10] ATLAS Collaboration, JHEP 10 (2019) 265.

[11] ATLAS Collaboration, ATL-PHYS-PUB-2017-014 (2017).

[12] ATLAS Collaboration, ATLAS-CONF-2018-020, 04 Jun 2018.

[13] ATLAS Collaboration, JHEP 01 (2019) 016.

[14] W.-Y. Keung and G. Senjanović, Phys. Rev. Lett. 50 (1983) 1427.

[15] ATLAS Collaboration, Phys. Lett. B 798 (2019) 134942. 